\title{
On asymptotics of Banach space-valued Itô functionals of Brownian rough paths
}

\author{
Yuzuru Inahama ${ }^{1}$ and Hiroshi Kawabi ${ }^{2}$ \\ 1 Department of Mathematics, Graduate School of Science and Engineering, \\ Tokyo Institute of Technology \\ 2-12-1, Oh-okayama, Meguro-ku, Tokyo 152-8551, JAPAN \\ inahama@math.titech.ac.jp \\ 2 Faculty of Mathematics, Kyushu University \\ 6-10-1, Hakozaki, Higashi-ku, Fukuoka 812-8581, JAPAN \\ kawabi@math.kyushu-u.ac.jp \\ Dedicated to Professor Kiyosi Itô on the occasion of his 90th birthday
}

\begin{abstract}
In this paper, we discuss asymptotics for certain Banach spacevalued Itô functionals of Brownian rough paths based on the results of Inahama-Kawabi [10] and Inahama [9]. Our main tool is the Banach spacevalued rough path theory of T. Lyons. As examples, we deal with heat processes on loop spaces and solutions of the stochastic differential equations (SDEs) on M-type 2 Banach spaces.
\end{abstract}

\section{Introduction}

Let $(X, H, \mu)$ be an abstract Wiener space, i.e., $X$ is a real separable Banach space, $H$ is the Cameron-Martin space and $\mu$ is the Wiener measure on $X$. Let $Y$ be another real separable Banach space and $w:=\left(w_{t}\right)_{0 \leq t \leq 1}$ be the $X$ valued Brownian motion on a completed probability space $(\Omega, \mathcal{F}, \mathbb{P})$ associated with $\mu$. We denote by $L(X, Y)$ the space of bounded linear operators from $X$ to $Y$. In this paper, we consider a class of $Y$-valued Wiener functionals $X^{\varepsilon}:=\left(X_{t}^{\varepsilon}\right)_{0 \leq t \leq 1}$ defined through the following formal Stratonovich type stochastic differential equation (SDE) on $Y$ :

$$
d X_{t}^{\varepsilon}=\sigma\left(X_{t}^{\varepsilon}\right) \circ \varepsilon d w_{t}+b\left(\varepsilon, X_{t}^{\varepsilon}\right) d t, \quad X_{0}^{\varepsilon}=0,
$$

where the coefficients $\sigma$ and $b$ take values in $L(X, Y)$ and $Y$, respectively, with a suitable regularity condition. Here, we note that the equation (1) cannot be discussed through the usual theory of SDEs when $X$ and $Y$ are infinite dimensional Banach spaces, because the diffusion coefficient $\sigma$ takes values in $L(X, Y)$. See Section 3 for the precise formulation of our Wiener 
functionals $X^{\varepsilon}$. The main objective of this paper is to discuss the FreidlinWentzell type large deviation principle for $X^{\varepsilon}$ and the asymptotic behavior of the Laplace type functional integral $\mathbb{E}\left[\exp \left(-F\left(X^{\varepsilon}\right) / \varepsilon^{2}\right)\right]$ as $\varepsilon \searrow 0$, which is called Laplace's method. For a class of continuous loop space-valued diffusion processes called heat processes, these asymptotics were studied in earlier papers Inahama-Kawabi [10] and Inahama [9], respectively. In this paper, we interpret our Wiener functionals $X^{\varepsilon}$ as Itô functionals of Brownian rough paths, and show that these asymptotics hold for wider classes of (infinite dimensional) Banach space-valued Wiener functionals by using the fact that the rough path theory of T. Lyons works on any Banach space.

To establish the large deviation principle for $X^{\varepsilon}$, due to the lack of the continuity of the Itô map $w \mapsto X^{\varepsilon}$, Schilder's theorem and the contraction principle may not be used directly. To overcome this difficulty, Freidlin and Wentzell developed refined techniques involving the exponential continuity (see Deuschel-Stroock [7]). On the other hand, recently, Ledoux-Qian-Zhang [16] gave a new proof for the large deviation principle by using the rough path theory. The basic idea in [16] is summarized as follows: First, they show that the laws of Brownian rough paths satisfy the large deviation principle. Next, they use the contraction principle since the Itô map is continuous in the framework of the rough path theory. Hence their approach seems straightforward and much simpler than conventional proofs. In [10], it is shown that their approach is also applicable to a class of stochastic processes on infinite dimensional spaces.

As an application of the large deviation principle, Laplace's method is investigated in many research fields of probability theory and mathematical physics. In finite dimensional settings, Schilder [19] initiated the study in the case of $X^{\varepsilon}=\varepsilon w$ and Azencott [2] and Ben Arous [3] continued this study for (1). (For results concerning with more general Wiener functionals, see Kusuoka-Stroock [13], [14] and Takanobu-Watanabe [20].) In these papers, the stochastic Taylor expansion for $X^{\varepsilon}$ plays an important role. The problem of [19] is rather easier because each term of the expansion is continuous, which comes from the fact that $X^{\varepsilon}$ is nothing but the scaled Brownian motion. So, there is no ambiguity in the formulation. However, in general, it is very complicated to give a precise interpretation on each term of this expansion through conventional stochastic analysis because the Itô map is not a continuous Wiener functional. On the other hand, Aida [1] proposed a new approach with the rough path theory for this problem recently. In [1], he obtained the stochastic Taylor expansion with respect to the topology of the space of geometric rough paths for finite dimensional cases. Since the Itô map is continuous in the rough path sense, each term of the expansion is continuous. Hence we do not need to face the difficulty mentioned above. Based on the idea of [1], the first author [9] showed the stochastic Taylor expansion in an infinite dimensional setting.

The organization of this paper is as follows: In Section 2, we give a simple review of the rough path theory and review the Cameron-Martin theorem and 
Fernique's theorem in the framework of Brownian rough paths. In Section 3, we give a framework and state our results. In Section 4, we give an outline of the proof of our results based on [10] and [9]. Finally, in Section 5, we give two examples to which our results are applicable. The first example is a class of heat processes described above and the second one comes from the SDE theory on M-type 2 Banach spaces.

\section{Preliminaries from the rough path theory}

In this section we set notations and review some basic results of the rough path theory.

First we recall the definition of spaces of geometric rough paths. Let $B$ be a real separable Banach space. The algebraic tensor product is denoted by $B \otimes_{\mathrm{a}} B$. We consider a norm $|\cdot|$ on $B \otimes_{\mathrm{a}} B$ such that $|x \otimes y| \leq|x|_{B} \cdot|y|_{B}$ holds for all $x, y \in B$. We denote by $B \otimes B$ the completion of $B \otimes_{\mathrm{a}} B$ by this norm. We often suppress the subscripts of Banach norms when there is no fear of confusion.

Let $2<p<3$ be the roughness and fix it throughout this paper. A continuous map $\bar{x}=\left(1, \bar{x}_{1}, \bar{x}_{2}\right)$ from the simplex $\Delta:=\{(s, t) \mid 0 \leq s \leq t \leq 1\}$ to the truncated tensor algebra $T^{(2)}(B):=\mathbb{R} \oplus B \oplus(B \otimes B)$ is said to be a $B$-valued rough path of roughness $p$ if it satisfies that, for every $s \leq u \leq t$,

$$
\begin{aligned}
& \bar{x}_{1}(s, t)=\bar{x}_{1}(s, u)+\bar{x}_{1}(u, t), \\
& \bar{x}_{2}(s, t)=\bar{x}_{2}(s, u)+\bar{x}_{2}(u, t)+\bar{x}_{1}(s, u) \otimes \bar{x}_{1}(u, t)
\end{aligned}
$$

and

$$
\left\|\bar{x}_{j}\right\|_{p / j}:=\left(\sup _{D} \sum_{l=1}^{n}\left|\bar{x}_{j}\left(t_{l-1}, t_{l}\right)\right|^{p / j}\right)^{j / p}<\infty \quad \text { for } j=1,2,
$$

where $D=\left\{0=t_{0}<t_{1}<\cdots<t_{n}=1\right\}$ runs over all finite partition of $[0,1]$. For two rough paths $\bar{x}$ and $\bar{y}, p$-variation distance is defined by

$$
d_{p}(\bar{x}, \bar{y})=\left\|\bar{x}_{1}-\bar{y}_{1}\right\|_{p}+\left\|\bar{x}_{2}-\bar{y}_{2}\right\|_{p / 2} .
$$

Let $P(B):=\left\{x \in C([0,1], B) \mid x_{0}=0\right\}$. For $x \in P(B)$, we denote by $\|x\|_{P(B)}:=\sup _{0 \leq t \leq 1}\left|x_{t}\right|_{B}$ and sometimes write $x(t)$ for $x_{t}$. Moreover we often write $\bar{x}_{1}(\cdot)$ for $\bar{x}_{1}(0, \cdot) \in P(B)$ for simplicity. We denote by $\mathrm{BV}(B):=$ $\left\{\gamma \in P(B) \mid\|\gamma\|_{1}<\infty\right\}$, where $\|\gamma\|_{1}$ denotes the total variation norm of $\gamma$. For $\gamma \in \mathrm{BV}(B)$, we set $\bar{\gamma}=\left(1, \bar{\gamma}_{1}, \bar{\gamma}_{2}\right)$ by

$$
\bar{\gamma}_{1}(s, t):=\gamma_{t}-\gamma_{s}, \quad \bar{\gamma}_{2}(s, t):=\int_{s}^{t}\left(\gamma_{u}-\gamma_{s}\right) \otimes d \gamma_{u}, \quad 0 \leq s \leq t \leq 1,
$$

where the right-hand side of $\bar{\gamma}_{2}$ is the Riemann-Stieltjes integral. A rough path obtained in this way is called the smooth rough path lying above $\gamma$. A 
rough path obtained as the $d_{p}$-limit of a sequence of smooth rough paths is called a geometric rough path and the set of all the geometric rough paths is denoted by $G \Omega_{p}(B)$. It is well-known that $G \Omega_{p}(B)$ is a complete separable metric space.

We set

$$
\mathcal{H}(B):=\left\{y \in P(B) \mid y_{t}=\int_{0}^{t} y_{s}^{\prime} d s \text { with }\|y\|_{\mathcal{H}(B)}^{2}:=\int_{0}^{1}\left|y_{t}^{\prime}\right|_{B}^{2} d t<\infty\right\} .
$$

Clearly, there are natural continuous injections $\mathcal{H}(B) \hookrightarrow \mathrm{BV}(B) \hookrightarrow G \Omega_{p}(B)$. Note that $\mathcal{H}(B)$ is dense in $G \Omega_{p}(B)$ and has a natural Hilbert structure in the case when $B$ is a Hilbert space.

Next we introduce Brownian rough paths on an abstract Wiener space $(X, H, \mu)$. Let $w=\left(w_{t}\right)_{t \geq 0}$ be the $X$-valued Brownian motion introduced in the previous section. For $\varepsilon>0$, the law of $\varepsilon w$ on $P(X)$ is denoted by $\mathbb{P}_{\varepsilon}^{\prime}$. Then $\left(P(X), \mathcal{H}(H), \mathbb{P}_{1}^{\prime}\right)$ is also an abstract Wiener space. We write $\mathcal{H}:=\mathcal{H}(H)$ for simplicity. When $|\cdot|_{X \otimes X}$ and $\mu$ satisfy the exactness condition (see Definition 1 in Ledoux-Lyons-Qian [15]), the Brownian rough path exists (see Theorem 3 in [15]). Let $\bar{w}=\left(1, \bar{w}_{1}, \bar{w}_{2}\right)$ be the Brownian rough path. It is the $\mathbb{P}$-almost sure limit of the $\overline{w(m)}$ as $m \rightarrow \infty$ in $G \Omega_{p}(X)$ with respect to $d_{p}$-topology, where $w(m)$ is the $m$-th dyadic polygonal approximation of $w$. Note that $\bar{w}_{1}(s, t)=w_{t}-w_{s}$ for $\mathbb{P}$-almost surely. We denote by $\mathbb{P}_{\varepsilon}, \varepsilon>0$, the law of the scaled Brownian rough path $\overline{\varepsilon w}=\left(1, \varepsilon \bar{w}_{1}, \varepsilon^{2} \bar{w}_{2}\right)$.

Now we present a theorem of Fernique for Brownian rough paths. We set $\xi(\bar{x}):=\left\|\bar{x}_{1}\right\|_{p}+\left\|\bar{x}_{2}\right\|_{p / 2}^{1 / 2}$. The following theorem is taken from Theorem 2.2 in $[9]$.

Theorem 2.1 There exists a positive constant $\beta$ such that

$$
\mathbb{E}\left[\exp \left(\beta \xi^{2}\right)\right]=\int_{G \Omega_{p}(X)} \exp \left(\beta \xi(\bar{w})^{2}\right) \mathbb{P}_{1}(d \bar{w})<\infty .
$$

Finally, we give a theorem for absolute continuity of the laws of shifted Brownian rough paths. It is similar to the well-known Cameron-Martin theorem. For $\bar{x} \in G \Omega_{p}(X)$ and $\gamma \in \mathrm{BV}(X)$, we define the shifted rough path $\overline{x+\gamma} \in G \Omega_{p}(X)$ by

$$
\begin{aligned}
\overline{(x+\gamma)}_{1}(s, t)=\bar{x}_{1}(s, t) & +\gamma_{t}-\gamma_{s}, \\
\overline{(x+\gamma)}_{2}(s, t)=\bar{x}_{2}(s, t)+ & \int_{s}^{t} \bar{x}_{1}(s, u) \otimes d \gamma_{u} \\
& +\int_{s}^{t}\left(\gamma_{u}-\gamma_{s}\right) \otimes \bar{x}_{1}(s, d u)+\bar{\gamma}_{2}(s, t) .
\end{aligned}
$$

Here the second and the third terms on the right-hand side are Young integrals. It is well-known that the map $(\bar{x}, \gamma) \mapsto \overline{x \pm \gamma}$ is continuous from $G \Omega_{p}(X) \times \mathrm{BV}(X)$ to $G \Omega_{p}(X)$ (see Theorem 3.3.2 in [17]). The following theorem is taken from Lemma 2.3 in [9]. 
Theorem 2.2 Let $\varepsilon>0$ and $h \in \mathcal{H}$. Then for every bounded measurable function $F$ on $G \Omega_{p}(X)$, it holds that

$$
\begin{aligned}
& \int_{G \Omega_{p}(X)} F(\overline{w+h}) \mathbb{P}_{\varepsilon}(d \bar{w}) \\
& =\int_{G \Omega_{p}(X)} F(\bar{w}) \exp \left(\frac{1}{\varepsilon^{2}} \int_{0}^{1} h^{\prime}(t) d \bar{w}_{1}(t)-\frac{1}{2 \varepsilon^{2}}\|h\|_{\mathcal{H}}^{2}\right) \mathbb{P}_{\varepsilon}(d \bar{w}),
\end{aligned}
$$

where $\int_{0}^{1} h^{\prime}(t) d \bar{w}_{1}(t)$ is the stochastic integral with respect to the scaled Brownian motion $\left(\bar{w}_{1}(0, t)\right)_{0 \leq t \leq 1}$ defined on the probability space $\left(G \Omega_{p}(X), \mathbb{P}_{\varepsilon}\right)$. (Hereafter we denote it $\bar{b} y[h](\bar{w})$ for simplicity.)

\section{Framework and results}

In this section, we set notations, introduce our Wiener functionals through the Itô map in the rough path sense and state our results. Throughout this paper, we only consider the projective norm on the tensor product of any pair of Banach spaces, and we always assume the exactness condition for $|\cdot| X \otimes X$ and $\mu$ to treat Brownian rough paths.

First, we set notations for coefficients. Let $\sigma \in C_{b}^{4}(Y, L(X, Y))$ and $b_{1}, b_{2} \in$ $C_{b}^{4}(Y, Y)$. We set $\tilde{X}:=X \oplus \mathbb{R}^{2}$ and define $\tilde{\sigma} \in C_{b}^{4}(Y, L(\tilde{X}, Y))$ by

$\tilde{\sigma}(y)[(x, u)]_{\tilde{X}}:=\sigma(y) x+b_{1}(y) u_{1}+b_{2}(y) u_{2}, \quad y \in Y, x \in X, u=\left(u_{1}, u_{2}\right) \in \mathbb{R}^{2}$.

Next, we consider the following differential equation in the rough path sense:

$$
d y_{t}=\tilde{\sigma}\left(y_{t}\right) d \tilde{x}_{t}, \quad y_{0}=0 .
$$

Then for any $\overline{\tilde{x}} \in G \Omega_{p}(\tilde{X})$, there exists a unique solution $\bar{z} \in G \Omega_{p}(\tilde{X} \oplus Y)$ in the rough path sense. Note that the natural projection of $\bar{z}$ onto the first component is $\bar{x}$. Projection of $\bar{z}$ onto the second component is denoted by $\bar{y} \in G \Omega_{p}(Y)$. We write $\bar{y}=\Phi(\overline{\tilde{x}})$ and call it a (unique) solution of (2). The map $\Phi: G \Omega_{p}(\tilde{X}) \rightarrow G \Omega_{p}(Y)$ is called the Itô map and is locally Lipschitz continuous in the sense of Theorem 6.2.2 in [17]. If $\tilde{x}_{t}=\left(\gamma_{t}, \lambda_{t}^{(1)}, \lambda_{t}^{(2)}\right)$ is a $\tilde{X}$-valued continuous path of finite variation, the map $t \mapsto \Phi(\bar{x})_{1}(0, t)$ is the solution of

$$
d y_{t}=\sigma\left(y_{t}\right) d \gamma_{t}+b_{1}\left(y_{t}\right) d \lambda_{t}^{(1)}+b_{2}\left(y_{t}\right) d \lambda_{t}^{(2)}, \quad y_{0}=0
$$

in the usual sense and $\bar{z}$ is the smooth rough path lying above $\left(\tilde{x}, \Phi(\overline{\tilde{x}})_{1}(0, \cdot)\right)$.

For $\lambda=\left(\lambda^{(1)}, \lambda^{(2)}\right) \in \mathrm{BV}\left(\mathbb{R}^{2}\right)$ and $\bar{x} \in G \Omega_{p}(X)$, we set $\iota(\bar{x}, \lambda) \in G \Omega_{p}(\tilde{X})$ by $\iota(\bar{x}, \lambda)_{1}(s, t)=\left(\bar{x}_{1}(s, t), \lambda_{t}-\lambda_{s}\right)$ and

$$
\begin{aligned}
\iota(\bar{x}, \lambda)_{2}(s, t)=\left(\bar{x}_{2}(s, t)\right. & \int_{s}^{t} \bar{x}_{1}(s, u) \otimes d \lambda_{u}, \\
& \left.\int_{s}^{t}\left(\lambda_{u}-\lambda_{s}\right) \otimes \bar{x}_{1}(s, d u), \int_{s}^{t}\left(\lambda_{u}-\lambda_{s}\right) \otimes d \lambda_{u}\right) .
\end{aligned}
$$


Here the second and the third component are Young integrals. If $\bar{h}$ is a smooth rough path lying above $h \in \mathrm{BV}(X)$, then $\iota(\bar{h}, \lambda)$ is a smooth rough path lying above $(h, \lambda) \in \mathrm{BV}(\tilde{X})$. Note that the map $\iota: G \Omega_{p}(X) \times \mathrm{BV}\left(\mathbb{R}^{2}\right) \rightarrow G \Omega_{p}(\tilde{X})$ is continuous. Here we also regard the Itô map defined above as a map from $\mathcal{H}(X)$ to $\mathcal{H}(Y)$. We define $\Psi_{\varepsilon}: \mathcal{H}(X) \rightarrow \mathcal{H}(Y)$ by $\Psi_{\varepsilon}(h)_{t}:=\Phi\left(\iota\left(\bar{h}, \lambda^{\varepsilon}\right)\right)_{1}(0, t)$ for $0 \leq t \leq 1$. That is, $y:=\Psi_{\varepsilon}(h)$ is the unique solution of

$$
d y_{t}=\sigma\left(y_{t}\right) d h_{t}+b_{1}\left(y_{t}\right) \varepsilon^{2} d t+b_{2}\left(y_{t}\right) d t, \quad y_{0}=0 .
$$

For the $X$-valued Brownian motion $w$, let $\bar{w}$ be the Brownian rough path over $X$. For $\varepsilon \geq 0$, we define a Wiener functional $X^{\varepsilon} \in P(Y)$ by

$$
X_{t}^{\varepsilon}:=\Phi\left(\iota\left(\overline{\varepsilon w}, \lambda^{\varepsilon}\right)\right)_{1}(0, t), \quad 0 \leq t \leq 1 .
$$

We investigate the asymptotic behavior of the law of $X^{\varepsilon}$ as $\varepsilon \searrow 0$. First, we state a large deviation principle which is essentially shown in Theorem 4.9 of Inahama-Kawabi [10].

Theorem 3.1 For $\varepsilon>0$, we denote by $\mathcal{V}_{\varepsilon}$ the law of the process $X^{\varepsilon}$. Then, $\left\{\mathcal{V}_{\varepsilon}\right\}_{\varepsilon>0}$ satisfies a large deviation principle as $\varepsilon \searrow 0$ with the good rate function $I$, where

$$
I(\phi)= \begin{cases}\frac{1}{2} \inf \left\{\|\gamma\|_{\mathcal{H}}^{2} \mid \phi=\Psi_{0}(\gamma)\right\}, & \text { if } \phi=\Psi_{0}(\gamma) \text { for some } \gamma \in \mathcal{H}, \\ \infty, & \text { otherwise. }\end{cases}
$$

More precisely, for any measurable set $K \subset P(Y)$, it holds that

$$
-\inf _{\phi \in K^{\circ}} I(\phi) \leq \liminf _{\varepsilon \searrow 0} \varepsilon^{2} \log \mathcal{V}_{\varepsilon}(K) \leq \limsup _{\varepsilon \searrow 0} \varepsilon^{2} \log \mathcal{V}_{\varepsilon}(K) \leq-\inf _{\phi \in \bar{K}} I(\phi) .
$$

As a consequence of Theorem 3.1, we have the following asymptotics for every bounded continuous function $F$ on $P(Y)$ :

$$
\lim _{\varepsilon \backslash 0} \varepsilon^{2} \log \mathbb{E}\left[\exp \left(-F\left(X^{\varepsilon}\right) / \varepsilon^{2}\right)\right]=-\inf \{F(\phi)+I(\phi) \mid \phi \in P(Y)\} .
$$

This is Varadhan's integral lemma. See [7] for example. Our next concern is to investigate the exact asymptotics of the integral on the left-hand side of above quality, i.e., to find the asymptotics behavior of $\mathbb{E}\left[\exp \left(-F\left(X^{\varepsilon}\right) / \varepsilon^{2}\right)\right]$ as $\varepsilon \searrow 0$.

In this paper, we impose the following assumptions on the function $F$. In what follows, we especially denote by $D$ the Fréchet derivatives on $\mathcal{H}(X)$ and $P(Y)$.

(F1): $F$ is a real-valued bounded continuous function defined on $P(Y)$.

(F2): The function $F \circ \Psi_{0}+\|\cdot\|_{\mathcal{H}}^{2} / 2$ defined on $\mathcal{H}$ attains its minimum 0 at a unique point $\gamma_{0} \in \mathcal{H}$. For this $\gamma_{0}$, we write $\phi_{0}:=\Psi_{0}\left(\gamma_{0}\right)$.

(F3): $F$ is three times Fréchet differentiable on a neighborhood $B\left(\phi_{0}\right)$ of $\phi_{0}$, 
and $D^{i} F, i=1,2,3$, are bounded on $B\left(\phi_{0}\right) \subset P(Y)$.

(F4): We consider the Hessian $A:=\left.D^{2}\left(F \circ \Psi_{0}\right)\left(\gamma_{0}\right)\right|_{\mathcal{H} \times \mathcal{H}}$ at the point $\gamma_{0} \in \mathcal{H}$. As a bounded self-adjoint operator on $\mathcal{H}$, the operator $A$ is strictly larger than $-\mathrm{Id}_{\mathcal{H}}$ in the form sense. (By the min-max principle, it is equivalent to assume that all eigenvalues of $A$ are strictly larger than -1.)

Now we are in a position to state our main result which is essentially due to Inahama [9]. The explicit value of $\alpha_{0}$ will be given later (Theorem 4.9) since we need to introduce a few more notations which we cannot introduce briefly.

Theorem 3.2 Let $X^{\varepsilon}$ be as above and assume (F1), (F2), (F3) and (F4). Then there exists a positive constant $\alpha_{0}$ such that

$$
\lim _{\varepsilon \searrow 0} \mathbb{E}\left[\exp \left(-F\left(X^{\varepsilon}\right) / \varepsilon^{2}\right)\right]=\alpha_{0} .
$$

Remark 3.3 As a continuation of this paper, we have already established the following asymptotic expansion formula:

$$
\mathbb{E}\left[\exp \left(-F\left(X^{\varepsilon}\right) / \varepsilon^{2}\right)\right]=\alpha_{0}+\alpha_{1} \varepsilon+\cdots+\alpha_{n} \varepsilon^{n}+O\left(\varepsilon^{n+1}\right), \quad n \in \mathbb{N} .
$$

The reader is referred to Inahama-Kawabi [11] for details.

\section{Proof of results}

In this section, we show Theorems 3.1 and 3.2. Since the Itô map $\Phi$ : $G \Omega_{p}(\tilde{X}) \rightarrow G \Omega_{p}(Y)$ is continuous, Theorem 3.1 is easily obtained by combining the contraction principle with the following Schilder type large deviations for the scaled Brownian rough path $\overline{\varepsilon w}$. The following result is taken from Theorem 3.2 in [10].

Theorem 4.1 For $\varepsilon>0$, we denote by $\mathbb{P}_{\varepsilon}$ the law of the scaled Brownian rough path $\overline{\varepsilon w}$ on $G \Omega_{p}(X)$. Then, $\left\{\mathbb{P}_{\varepsilon}\right\}_{\varepsilon>0}$ satisfies a large deviation principle as $\varepsilon \searrow 0$ with the good rate function $I_{0}$, where

$$
I_{0}(\bar{x})= \begin{cases}\frac{1}{2}\|h\|_{\mathcal{H}}^{2}, & \text { if } \bar{x}=\bar{h} \text { for some } h \in \mathcal{H}, \\ \infty, & \text { otherwise. }\end{cases}
$$

More precisely, for any measurable set $K \subset G \Omega_{p}(X)$, it holds that

$$
\begin{aligned}
-\inf _{\bar{x} \in K^{\circ}} I_{0}(\bar{x}) & \leq \liminf _{\varepsilon \searrow 0} \varepsilon^{2} \log \mathbb{P}_{\varepsilon}(K) \\
& \leq \limsup _{\varepsilon \searrow 0} \varepsilon^{2} \log \mathbb{P}_{\varepsilon}(K) \leq-\inf _{\bar{x} \in \bar{K}} I_{0}(\bar{x}) .
\end{aligned}
$$

In the sequel, we give an outline of the proof of Theorem 3.2 based on the arguments in [9]. We divide into several subsections. 


\subsection{Stochastic Taylor expansion in the sense of rough paths}

In this subsection, we introduce the stochastic Taylor expansion for the differential equation (3) in the sense of rough paths. We remark that it is deterministic in this case. Hence the term "stochastic Taylor expansion" may not be appropriate anymore. In the sequel, we denote by $\nabla$ the Fréchet derivative on $Y$.

Let $\gamma \in \mathcal{H}(X)$ and $\phi:=\Psi_{0}(\gamma)$. For each $h \in \mathcal{H}(X)$, we define $\chi_{t}=\chi(h)_{t}$ and $\psi_{t}=\psi(h, h)_{t}$ by

$$
d \chi_{t}-(\nabla \sigma)\left(\phi_{t}\right)\left[\chi_{t}, d \gamma_{t}\right]-\left(\nabla b_{2}\right)\left(\phi_{t}\right)\left[\chi_{t}\right] d t=\sigma\left(\phi_{t}\right) d h_{t}, \quad \chi_{0}=0,
$$

and

$$
\begin{aligned}
& d \psi_{t}-(\nabla \sigma)\left(\phi_{t}\right)\left[\psi_{t}, d \gamma_{t}\right]-\left(\nabla b_{2}\right)\left(\phi_{t}\right)\left[\psi_{t}\right] d t \\
& =2(\nabla \sigma)\left(\phi_{t}\right)\left[\chi_{t}, d h_{t}\right]+\left(\nabla^{2} \sigma\right)\left(\phi_{t}\right)\left[\chi_{t}, \chi_{t}, d \gamma_{t}\right]+\left(\nabla^{2} b_{2}\right)\left(\phi_{t}\right)\left[\chi_{t}, \chi_{t}\right] d t, \psi_{0}=0,
\end{aligned}
$$

where $\nabla^{i} \sigma: Y \rightarrow L^{i}(Y, \ldots, Y ; L(X, Y)), \nabla^{i} b_{2}: Y \rightarrow L^{i}(Y, \ldots, Y ; Y)$ for $i=1,2$. Here $L^{i}\left(B_{1}, \ldots, B_{i} ; B_{i+1}\right)$ denotes the space of bounded multi-linear maps from the product of Banach spaces $B_{1} \times \cdots \times B_{i}$ to another Banach space $B_{i+1}$. All Fréchet derivatives on (5) and (6) exist and bounded. We should note that $\chi=D \Psi_{0}(\gamma)[h]$ and $\psi=D^{2} \Psi_{0}(\gamma)[h, h]$ hold, where $\Psi_{0}: \mathcal{H}(X) \rightarrow \mathcal{H}(Y)$ is defined in Section 3.

At the beginning, we give a simple lemma to deal with differential equations such as (5) and (6). See Lemma 3.1 in [9] for details.

Lemma 4.2 Fix $\gamma \in \mathcal{H}(X)$ and $\phi=\Psi_{0}(\gamma)$. Let $M:[0,1] \rightarrow L(Y, Y)$ be the solution of the differential equation

$$
d M_{t}=d \Omega_{t} M_{t}, \quad M_{0}=\mathrm{Id}_{Y},
$$

where

$$
d \Omega_{t}:=(\nabla \sigma)\left(\phi_{t}\right)\left[\cdot, d \gamma_{t}\right]+\left(\nabla b_{2}\right)\left(\phi_{t}\right)[\cdot] d t \in L(Y, Y), \quad t \geq 0
$$

Then $M_{t}$ is invertible for all $t \geq 0$.

Moreover, for each $k \in \mathcal{H}(Y)$, we define $\Gamma(k)=\Gamma_{\gamma}(k) \in \mathcal{H}(Y)$ by

$$
\Gamma(k)_{t}:=M_{t} \int_{0}^{t} M_{s}^{-1} d k_{s}, \quad t \geq 0 .
$$

Then $\Gamma(k)$ is the unique solution of the differential equation

$$
d \Gamma(k)_{t}-d \Omega_{t} \Gamma(k)_{t}=d k_{t}, \quad \Gamma(k)_{0}=0,
$$

and the operator $\Gamma: \mathcal{H}(Y) \rightarrow \mathcal{H}(Y)$ can be extended to a bounded linear operator from $P(Y)$ to $P(Y)$. 
By using Lemma 4.2, we have the following expressions for the solutions of the equations (5) and (6):

$$
\chi(h)_{t}=\Gamma\left(\int_{0}^{.} \sigma\left(\phi_{s}\right) d h_{s}\right)_{t}, \quad \psi(h, h)_{t}=\Gamma\left(\int_{0} d C_{h, h}(s)\right)_{t},
$$

where

$$
\begin{aligned}
d C_{h, \hat{h}}(s)= & (\nabla \sigma)\left(\phi_{s}\right)\left[\chi(h)_{s}, d \hat{h}_{s}\right]+(\nabla \sigma)\left(\phi_{s}\right)\left[\chi(\hat{h})_{s}, d h_{s}\right] \\
& +\left(\nabla^{2} \sigma\right)\left(\phi_{s}\right)\left[\chi(h)_{s}, \chi(\hat{h})_{s}, d \gamma_{s}\right] \\
& +\left(\nabla^{2} b_{2}\right)\left(\phi_{s}\right)\left[\chi(h)_{s}, \chi(\hat{h})_{s}\right] d s \quad \text { for } h, \hat{h} \in \mathcal{H}(X) .
\end{aligned}
$$

Next we give estimates of $\sup _{0 \leq t \leq 1}|\chi(h)|_{Y}$ and $\sup _{0 \leq t \leq 1}\left|\psi(h, h)_{t}\right|_{Y}$ in terms of the function $\xi$. See Lemmas 5.1 and 5.3 in [9] for the proof.

Lemma 4.3 Let $\chi_{t}=\chi(h)_{t}$ and $\psi_{t}=\psi(h, h)_{t}$. Let $r_{0}, r_{1}$ be any positive constants. Then, there exists a positive constant $c=c\left(r_{0}, r_{1}\right)$ such that

$$
\begin{aligned}
& \sup _{0 \leq t \leq 1}\left|\chi(h)_{t}\right|_{Y} \leq c \xi(\bar{h}), \\
& \sup _{0 \leq t \leq 1}\left|\psi(h, h)_{t}\right|_{Y} \leq c \xi(\bar{h})^{2}
\end{aligned}
$$

hold for all $h \in \mathcal{H}(X)$ with $\xi(\bar{h}) \leq r_{0}$ and for all $\gamma \in \mathcal{H}(X)$ with $\|\gamma\|_{\mathcal{H}(X)} \leq r_{1}$. Moreover the maps $h \in \mathcal{H}(X) \mapsto \chi(h)$ and $h \in \mathcal{H}(X) \mapsto \psi(h, h)$ can be extended to continuous maps from $G \Omega_{p}(X)$ to $P(Y)$.

By the above lemma, we can define $\chi(\bar{w})$ and $\psi(\bar{w}, \bar{w})$ by the continuous extensions of $\chi(h)$ and $\psi(h, h)$, respectively. From now, we aim to give an explicit representation of $\psi(\bar{w}, \bar{w})$. We set some notations. For $K \in L^{2}(X, X ; Y)$, we define the trace of $K$ by

$$
\operatorname{Tr}(K):=\int_{X} K[x, x] \mu(d x) .
$$

By virtue of Fernique's theorem (Theorem 3.1 in Kuo [12]), it holds that

$$
|\operatorname{Tr}(K)|_{Y} \leq\|K\|_{L^{2}(X, X ; Y)} \cdot \int_{X}|x|_{X}^{2} \mu(d w)<\infty .
$$

This means that $\operatorname{Tr}: L^{2}(X, X ; Y) \rightarrow Y$ is a bounded linear map. By recalling Itô-Nisio's theorem, we have

$$
\lim _{n \rightarrow \infty}\left|\operatorname{Tr}(K)-\sum_{i=1}^{n} K\left[e_{i}, e_{i}\right]\right|_{Y}=0,
$$

where $\left\{e_{i}\right\}_{i=1}^{\infty} \subset X^{*}$ is a C.O.N.S. of $H$. We denote $Q_{2}\left(\phi_{s}\right) \in L(X, X ; Y)$, $0 \leq s \leq 1$, by 


$$
Q_{2}\left(\phi_{s}\right)\left[x_{1}, x_{2}\right]:=\frac{1}{2}(\nabla \sigma)\left(\phi_{s}\right)\left[\sigma\left(\phi_{s}\right) x_{1}, x_{2}\right]_{Y \times X}, \quad x_{1}, x_{2} \in X .
$$

For $\alpha \in Y^{*}, 0 \leq t \leq 1$ and $(u, s) \in \Delta_{t}:=\{(u, s) \mid 0 \leq u \leq s \leq t\}$, we define a continuous map $K(\alpha)_{t}(u, s): \Delta_{t} \rightarrow L^{2}(X, X ; \mathbb{R})$ by

$$
\begin{aligned}
& K(\alpha)_{t}(u, s)\left[x_{1}, x_{2}\right]_{X \times X} \\
& :=\alpha\left(M_{t} M_{s}^{-1}(\nabla \sigma)\left(\phi_{s}\right)\left[M_{s} M_{u}^{-1} \sigma\left(\phi_{u}\right) x_{1}, x_{2}\right]_{Y \times X}\right), \quad x_{1}, x_{2} \in X .
\end{aligned}
$$

Then we have

Lemma 4.4 Let $\psi=\psi(\bar{w}, \bar{w})$ be the continuous extension of $\psi=\psi(h, h)$ as in Lemma 4.3. Then for any $\alpha \in Y^{*}$ and $t \in[0,1]$,

$$
\begin{aligned}
& \alpha\left(\psi(\bar{w}, \bar{w})_{t}\right) \\
& =2 \int_{0}^{t} \int_{0}^{s} K(\alpha)_{t}(u, s)\left[d \bar{w}_{1}(u), d \bar{w}_{1}(s)\right]+2 \alpha\left(\Gamma\left(\int_{0}^{\cdot} \operatorname{Tr}\left(Q_{2}\right)\left(\phi_{s}\right) d s\right)_{t}\right) \\
& \quad+\alpha\left(\Gamma\left(\int_{0}\left(\nabla^{2} \sigma\right)\left(\phi_{s}\right)\left[\chi(\bar{w})_{s}, \chi(\bar{w})_{s}, d \gamma_{s}\right]+\left(\nabla^{2} b_{2}\right)\left(\phi_{s}\right)\left[\chi(\bar{w})_{s}, \chi(\bar{w})_{s}\right] d s\right)_{t}\right)
\end{aligned}
$$

holds $\mathbb{P}_{1}$-almost surely $\bar{w}$, where the first term on the right-hand side is a usual stochastic iterated integral with respect to the Brownian motion $\left(\bar{w}_{1}(0, t)\right)_{0 \leq t \leq 1}$ on the probability space $\left(G \Omega_{p}(X), \mathbb{P}_{1}\right)$.

Proof. By (7) and (8), we have the following expression for every $h \in \mathcal{H}(X)$ :

$$
\begin{aligned}
\psi(h, h)_{t} & \\
= & 2 \Gamma\left(\int_{0}^{.}(\nabla \sigma)\left(\phi_{s}\right)\left[\chi(h)_{s}, d h_{s}\right]\right)_{t} \\
& +\Gamma\left(\int_{0}^{r}\left(\nabla^{2} \sigma\right)\left(\phi_{s}\right)\left[\chi(h)_{s}, \chi(h)_{s}, d \gamma_{s}\right]+\left(\nabla^{2} b_{2}\right)\left(\phi_{s}\right)\left[\chi(h)_{s}, \chi(h)_{s}\right] d s\right)_{t} \\
= & 2 \int_{0}^{t} \int_{0}^{s} M_{t} M_{s}^{-1}(\nabla \sigma)\left(\phi_{s}\right)\left[M_{s} M_{u}^{-1} \sigma\left(\phi_{u}\right) d h_{u}, d h_{s}\right]_{Y \times X} \\
& +\Gamma\left(\int_{0}^{r}\left(\nabla^{2} \sigma\right)\left(\phi_{s}\right)\left[\chi(h)_{s}, \chi(h)_{s}, d \gamma_{s}\right]+\left(\nabla^{2} b_{2}\right)\left(\phi_{s}\right)\left[\chi(h)_{s}, \chi(h)_{s}\right] d s\right)_{t} .
\end{aligned}
$$

Then for $\alpha \in Y^{*},(9)$ leads us that

$$
\begin{aligned}
& \alpha\left(\psi(\overline{w(m)}, \overline{w(m)})_{t}\right) \\
&=2 \int_{0}^{t} \int_{0}^{s} K(\alpha)_{t}(u, s) {[d w(m)(u), d w(m)(s)] } \\
&+\alpha\left(\Gamma \left(\int_{0}^{.}\left(\nabla^{2} \sigma\right)\left(\phi_{s}\right)\left[\chi(\overline{w(m)})_{s}, \chi(\overline{w(m)})_{s}, d \gamma_{s}\right]\right.\right. \\
&\left.\left.+\left(\nabla^{2} b_{2}\right)\left(\phi_{s}\right)\left[\chi(\overline{w(m)})_{s}, \chi(\overline{w(m)})_{s}\right] d s\right)_{t}\right) .
\end{aligned}
$$


Here we have the convergence

$$
\begin{aligned}
\lim _{m \rightarrow \infty} \mathbb{E}[ & \mid \int_{0}^{t} \int_{0}^{s} K(\alpha)_{t}(u, s)[d w(m)(u), d w(m)(s)] \\
& \left.-\left\{\int_{0}^{t} \int_{0}^{s} K(\alpha)_{t}(u, s)\left[d w_{u}, d w_{s}\right]+\frac{1}{2} \int_{0}^{t} \operatorname{Tr}\left(K(\alpha)_{t}(s, s)\right) d s\right\} \mid\right]=0
\end{aligned}
$$

and the equality

$$
\begin{aligned}
& \int_{0}^{t} \operatorname{Tr}\left(K(\alpha)_{t}(s, s)\right) d s \\
& =\alpha\left(M_{t} \int_{0}^{t} M_{s}^{-1} \operatorname{Tr}\left(Q_{2}\right)\left(\phi_{s}\right) d s\right)=\alpha\left(\Gamma\left(\int_{0}^{\cdot} \operatorname{Tr}\left(Q_{2}\right)\left(\phi_{s}\right) d s\right)_{t}\right) .
\end{aligned}
$$

Hence by letting $m \rightarrow \infty$ on both sides of (10) and by recalling Lemma 4.3 , (11) and (12), we obtain the desired assertion.

Now we are in a position to give the stochastic Taylor expansion up to the order 2. For fixed $\gamma \in \mathcal{H}(Y)$, we recall that $\phi \in \mathcal{H}(X)$ is defined by $\phi=\Psi_{0}(\gamma)$. For $0<\varepsilon \leq 1$ and $h \in \mathcal{H}(X), R_{\varepsilon}^{i}=R_{\varepsilon}^{i}(h), i=1,2,3$, are defined as follows:

$$
\begin{aligned}
& R_{\varepsilon}^{1}(t):=\Psi_{\varepsilon}(h+\gamma)_{t}-\phi_{t} \\
& R_{\varepsilon}^{2}(t):=\Psi_{\varepsilon}(h+\gamma)_{t}-\phi_{t}-\chi(h)_{t} \\
& R_{\varepsilon}^{3}(t):=\Psi_{\varepsilon}(h+\gamma)_{t}-\phi_{t}-\chi(h)_{t}-\frac{1}{2} \psi(h, h)_{t}-\varepsilon^{2} \Gamma\left(\int_{0}^{.} b_{1}\left(\phi_{s}\right) d s\right)_{t}
\end{aligned}
$$

Then we have the following estimates for the remainder terms of the stochastic Taylor expansion. See Lemma 6.1 in [9] for details.

Lemma 4.5 For $0<\varepsilon \leq 1$, let $R_{\varepsilon}^{1}(t), R_{\varepsilon}^{2}(t)$ and $R_{\varepsilon}^{3}(t)$ be as above. Let $r_{0}$ and $r_{1}$ be any positive constants. Then, there exists a positive constant $c=c\left(r_{0}, r_{1}\right)$ such that

$$
\sup _{0 \leq t \leq 1}\left|R_{\varepsilon}^{i}(t)\right|_{Y} \leq c(\xi(\bar{h})+\varepsilon)^{i}, \quad i=1,2,3
$$

hold for all $h \in \mathcal{H}(X)$ with $\xi(\bar{h}) \leq r_{0}$ and $\gamma \in \mathcal{H}(X)$ with $\|\gamma\|_{\mathcal{H}(X)} \leq r_{1}$. Moreover, for each fixed $\varepsilon$ and $\gamma$ the map $h \in \mathcal{H}(X) \mapsto R_{\varepsilon}^{i}=R_{\varepsilon}^{i}(h) \in$ $\mathcal{H}(Y), i=1,2,3$, can be extended to continuous maps from $G \Omega_{p}(X)$ to $P(Y)$.

\subsection{Computation of the Hessian}

In this subsection, we present some fundamental properties on the Hessian $A$ defined in Section 2. First, we give an explicit representation of $A$. We recall the equations (5), (6) and use Lemma 4.2. Then, for $h, \hat{h} \in \mathcal{H}$, we obtain 


$$
\begin{aligned}
& (A h, \hat{h})_{\mathcal{H}} \\
& =D F\left(\phi_{0}\right)\left[D^{2} \Psi_{0}\left(\gamma_{0}\right)[h, \hat{h}]\right]+D^{2} F\left(\phi_{0}\right)\left[D \Psi_{0}\left(\gamma_{0}\right)[h], D \Psi_{0}\left(\gamma_{0}\right)[\hat{h}]\right] \\
& =D F\left(\phi_{0}\right)\left[M \cdot \int_{0}^{\cdot} M_{s}^{-1} d C_{h, \hat{h}}(s)\right]+D^{2} F\left(\phi_{0}\right)[\chi(h), \chi(\hat{h})] .
\end{aligned}
$$

Here we set

$$
\begin{aligned}
V(h, \hat{h})_{t}:=M_{t} \int_{0}^{t} M_{s}^{-1}\{(\nabla \sigma) & \left(\phi_{0}(s)\right)\left[\chi(h)_{s}, d \hat{h}_{s}\right] \\
& \left.+(\nabla \sigma)\left(\phi_{0}(s)\right)\left[\chi(\hat{h})_{s}, d h_{s}\right]\right\}, \quad t \geq 0,
\end{aligned}
$$

and define a bounded self-adjoint operator $\tilde{A}$ on $\mathcal{H}$ by

$$
(\tilde{A} h, \hat{h})_{\mathcal{H}}:=D F\left(\phi_{0}\right)[V(h, \hat{h})] \quad \text { for } h, \hat{h} \in \mathcal{H} .
$$

Then by (13), (14) and (15), we obtain

$$
\begin{aligned}
& ((A-\tilde{A}) h, \hat{h})_{\mathcal{H}} \\
& =D F\left(\phi_{0}\right)\left[\Gamma \left(\int_{0}\left(\nabla^{2} \sigma\right)\left(\phi_{0}(s)\right)\left[\chi(h)_{s}, \chi(\hat{h})_{s}, d \gamma_{0}(s)\right]\right.\right. \\
& \left.\left.\quad+\left(\nabla^{2} b_{2}\right)\left(\phi_{0}(s)\right)\left[\chi(h)_{s}, \chi(\hat{h})_{s}\right] d s\right)\right]+D^{2} F\left(\phi_{0}\right)[\chi(h), \chi(\hat{h})]
\end{aligned}
$$

and it implies that

$$
\left|((A-\tilde{A}) h, \hat{h})_{\mathcal{H}}\right| \leq c\|\chi(h)\|_{P(Y)} \cdot\|\chi(\hat{h})\|_{P(Y)} \leq c\|h\|_{P(X)} \cdot\|\hat{h}\|_{P(X)}
$$

holds for some constant $c>0$. Then by applying Theorem 4.6 in [12] to an abstract Wiener space $\left(P(X), \mathcal{H}, \mathbb{P}_{1}^{\prime}\right)$, we can see that $A-\tilde{A}$ is a trace class operator on $\mathcal{H}$.

Moreover, we have the following properties on the operators $A$ and $\tilde{A}$ :

Lemma 4.6 (1) $A$ and $\tilde{A}$ are self-adjoint Hilbert-Schmidt operators on $\mathcal{H}$.

(2) The continuous extension of the quadratic form defined by $A-\tilde{A}$ is represented as

$$
\begin{aligned}
\langle(A-\tilde{A}) \bar{w}, \bar{w}\rangle= & D^{2} F\left(\phi_{0}\right)[\chi(\bar{w}), \chi(\bar{w})] \\
+D F\left(\phi_{0}\right)\left[\Gamma \left(\int_{0}\left(\nabla^{2} \sigma\right)\left(\phi_{0}(s)\right)\left[\chi(\bar{w})_{s}, \chi(\bar{w})_{s}, d \gamma_{0}(s)\right]\right.\right. & \left.\left.+\left(\nabla^{2} b_{2}\right)\left(\phi_{0}(s)\right)\left[\chi(\bar{w})_{s}, \chi(\bar{w})_{s}\right] d s\right)\right] .
\end{aligned}
$$

Proof. Firstly, we note that the unitary isometry $\mathcal{H} \cong L^{2}([0,1], \mathbb{R}) \otimes H$, where $\otimes$ denotes the Hilbert-Schmidt tensor product. Let $\left\{v_{j}^{\prime}\right\}_{j=0}^{\infty}$ be a C.O.N.S. of $L^{2}([0,1], \mathbb{R})$ defined by $v_{0}^{\prime}(t)=1, v_{2 j-1}^{\prime}(t)=\sqrt{2} \sin (2 \pi j t)$ and $v_{2 j}^{\prime}(t)=$ $\sqrt{2} \cos (2 \pi j t)$ for $j \in \mathbb{N}$. Secondly, let $\left\{e_{l}\right\}_{l=1}^{\infty}$ be a C.O.N.S. of $H$ such that 
$\sum_{l=1}^{\infty}\left|e_{l}\right|_{X}^{2}<\infty$. (See Theorem 3.5.10 in Bogachev [4].) Then $\left\{v_{j} \otimes e_{l}\right\}_{j, l=1}^{\infty}$ is a C.O.N.S. of $\mathcal{H}$. Hence by noting $\sum_{l=1}^{\infty}\left|e_{l}\right|_{X}^{2}<\infty$ and following the proof of Lemma 7.2 and Corollary 7.5 in [9], we can show the items (1) and (2).

Next, we consider the stochastic integration of the kernel associated with $\tilde{A}$. Recall any self-adjoint Hilbert-Schmidt operator $S$ on $\mathcal{H}$ corresponds to a kernel function $K_{S} \in L^{2}([0,1] \times[0,1], H \otimes H)$ with $K_{S}(u, s)=K_{S}(s, u)^{*}$ for almost all $(u, s)$ since $\mathcal{H} \cong L^{2}([0,1], H) \cong L^{2}([0,1], \mathbb{R}) \otimes H$. The correspondence $S \mapsto K_{S}$ is isometric. Then for the $X$-valued Brownian motion $w=\left(w_{t}\right)_{0 \leq t \leq 1}$, an iterated stochastic integral $\hat{K}_{S}(w):=2 \int_{0}^{1} \int_{0}^{s} K_{S}(u, s)\left[d w_{u}, d w_{s}\right]$ is welldefined. Clearly, this random variable is in $L^{2}\left(\mathbb{P}_{1}^{\prime}\right)$ with expectation 0 . The correspondence $S \mapsto \hat{K}_{S} \in L^{2}\left(\mathbb{P}_{1}^{\prime}\right)$ is isometric. The following lemma is essentially shown in Corollary 7.3 and Lemma 7.4 in [9].

Lemma 4.7 (1) For each $\alpha \in P(Y)^{*}, \alpha \circ V$ is a Hilbert-Schmidt symmetric bilinear form on $\mathcal{H}$. (We also denote by $\alpha \circ V$ the self-adjoint Hilbert-Schmidt operator on $\mathcal{H}$ associated with this bilinear form.)

(2) For any $\alpha \in P(Y)^{*}$, it holds that

$$
\alpha(\Theta(\bar{w}))=\hat{K}_{\alpha \circ V}\left(\bar{w}_{1}\right), \quad \mathbb{P}_{1} \text {-almost surely },
$$

where

$$
\begin{aligned}
\Theta(\bar{w}):= & \psi(\bar{w}, \bar{w})-\Gamma\left(\int_{0} \operatorname{Tr}\left(Q_{2}\right)\left(\phi_{0}(s)\right) d s\right) \\
& -\Gamma\left(\int_{0}\left(\nabla^{2} \sigma\right)\left(\phi_{0}(s)\right)\left[\chi(\bar{w})_{s}, \chi(\bar{w})_{s}, d \gamma_{0}(s)\right]\right. \\
& \left.+\left(\nabla^{2} b_{2}\right)\left(\phi_{0}(s)\right)\left[\chi(\bar{w})_{s}, \chi(\bar{w})_{s}\right] d s\right) .
\end{aligned}
$$

In particular, $D F(\phi)[\Theta(\bar{w})]=\hat{K}_{\tilde{A}}\left(\bar{w}_{1}\right)$ holds $\mathbb{P}_{1}$-almost surely.

Before closing this subsection, we present an integral formula to compute the quantity $\alpha_{0}$. See the proof of Lemma 8.3 in [9] for details.

Lemma 4.8 It holds that

$$
\begin{aligned}
\int_{G \Omega_{p}(X)} \exp \left\{-\frac{1}{2}\left(\hat{K}_{\tilde{A}}\left(\bar{w}_{1}\right)-\right.\right. & \langle(A-\tilde{A}) \bar{w}, \bar{w}\rangle)\} \mathbb{P}_{1}(d \bar{w}) \\
& =e^{-\frac{1}{2} \operatorname{Tr}(A-\tilde{A})} \cdot \operatorname{det}_{2}\left(\operatorname{Id}_{\mathcal{H}}+A\right)^{-1 / 2}
\end{aligned}
$$

where det $_{2}$ denotes the Carleman-Fredholm determinant.

\subsection{Outline of the proof of Theorem 3.2}

In this subsection, we explain about the outline of the proof of Theorem 3.2 briefly. Besides we give the explicit value of $\alpha_{0}$. For details, the reader is referred to Section 8 in [9]. 
We proceed with the following steps. First, we denote a neighborhood of $\overline{\gamma_{0}}$ and its exterior in $G \Omega_{p}(X)$ by $U\left(\overline{\gamma_{0}}\right)$ and $U\left(\overline{\gamma_{0}}\right)^{c}$, respectively. We divide our functional integral into

$$
\begin{aligned}
& \mathbb{E}\left[\exp \left(-F\left(X^{\varepsilon}\right) / \varepsilon^{2}\right)\right] \\
& =\int_{U\left(\overline{\gamma_{0}}\right)}+\int_{U\left(\overline{\gamma_{0}}\right)^{c}} \exp \left\{-\frac{1}{\varepsilon^{2}} F\left(\Phi\left(\iota\left(\bar{w}, \lambda^{\varepsilon}\right)\right)_{1}\right)\right\} \mathbb{P}_{\varepsilon}(d \bar{w})=: I_{1}(\varepsilon)+I_{2}(\varepsilon) .
\end{aligned}
$$

For the integral $I_{2}(\varepsilon)$, we can neglect as $\varepsilon \searrow 0$ by the large deviation principle for Brownian rough paths (Theorem 4.1). For the integral $I_{1}(\varepsilon)$, we regard $\Phi\left(\iota\left(\bar{w}, \lambda^{\varepsilon}\right)\right)_{1}$ as the perturbation of a quadratic function on $U\left(\overline{\gamma_{0}}\right)$.

Next, we put $\phi=\phi_{0}, \gamma=\gamma_{0}$ and consider

$$
\begin{aligned}
g_{\varepsilon}^{1}(h) & :=\chi(h), \\
g_{\varepsilon}^{2}(h) & :=\psi(h, h)+2 \varepsilon^{2} \Gamma\left(\int_{0} b_{1}\left(\phi_{0}(s)\right) d s\right), \\
R_{\varepsilon}^{3}\left(h-\gamma_{0}\right) & :=\Psi_{\varepsilon}(h)-\phi_{0}-g_{\varepsilon}^{1}\left(h-\gamma_{0}\right)-\frac{1}{2} g_{\varepsilon}^{2}\left(h-\gamma_{0}\right)
\end{aligned}
$$

for $\varepsilon>0$ and $h \in \mathcal{H}(X)$. Note that $\bar{w} \in G \Omega_{p}(X) \mapsto \Phi\left(\iota\left(\bar{w}, \lambda^{\varepsilon}\right)\right)_{1} \in P(Y)$ is the continuous extension of $h \in \mathcal{H}(X) \mapsto \Psi_{\varepsilon}(h) \in \mathcal{H}(Y)$. By recalling Lemmas 4.3 and 4.5, all functions on (18) can be extended to continuous functions on $G \Omega_{p}(X)$, which will be denoted by the same symbols. (For example, we write $g_{\varepsilon}^{1}\left(\overline{w-\gamma_{0}}\right)$, etc. $)$

Then by combining the Taylor expansion for $F$ and (18), we obtain that

$$
\begin{aligned}
& F\left(\Phi\left(\iota\left(\bar{w}, \lambda^{\varepsilon}\right)\right)_{1}\right)-F\left(\phi_{0}\right) \\
& =D F\left(\phi_{0}\right)\left[g_{\varepsilon}^{1}\left(\overline{w-\gamma_{0}}\right)\right] \\
& \quad+\frac{1}{2} D F\left(\phi_{0}\right)\left[g_{\varepsilon}^{2}\left(\overline{w-\gamma_{0}}\right)\right]+\frac{1}{2} D^{2} F\left(\phi_{0}\right)\left[g_{\varepsilon}^{1}\left(\overline{w-\gamma_{0}}\right), g_{\varepsilon}^{1}\left(\overline{w-\gamma_{0}}\right)\right] \\
& \quad+R_{\varepsilon}^{3}(F)\left(\overline{w-\gamma_{0}}\right),
\end{aligned}
$$

where $R_{\varepsilon}^{3}(F)\left(\overline{w-\gamma_{0}}\right)$ is the remainder term and all the functions above are continuous on $G \Omega_{p}(X)$.

On the other hand, by Assumption (F2), the function $h \in \mathcal{H} \mapsto F\left(\Psi_{0}(h)\right)+$ $\|h\|_{\mathcal{H}}^{2} / 2$ attains minimum 0 at $\gamma_{0} \in \mathcal{H}$. Hence for any $h \in \mathcal{H}$,

$$
0=\left(\gamma_{0}, h\right)_{\mathcal{H}}+D F\left(\phi_{0}\right)[\chi(h)]
$$

holds. As the continuous extension of the above equality, it holds that

$$
D F\left(\phi_{0}\right)\left[g_{\varepsilon}^{1}\left(\overline{w-\gamma_{0}}\right)\right]=\left\|\gamma_{0}\right\|_{\mathcal{H}}^{2}-\left[\gamma_{0}\right](\bar{w}), \quad \mathbb{P}_{\varepsilon} \text {-almost surely }
$$

Then by combining (19) with (20), and by using Lemmas 2.2, 4.6, 4.7, 4.8, we obtain 


$$
\begin{aligned}
\lim _{\varepsilon \searrow 0} I_{1}(\varepsilon) & \\
= & \int_{G \Omega_{p}(X)} \exp \left\{-\frac{1}{2}\left(D F\left(\phi_{0}\right)\left[g_{1}^{2}(\bar{w})\right]+D^{2} F\left(\phi_{0}\right)\left[g_{1}^{1}(\bar{w}), g_{1}^{1}(\bar{w})\right]\right)\right\} \mathbb{P}_{1}(d \bar{w}) \\
= & \exp \left\{-\frac{1}{2} \operatorname{Tr}(A-\tilde{A})-D F\left(\phi_{0}\right)\left[\Gamma\left(\int_{0} b_{1}\left(\phi_{0}(s)\right)+\operatorname{Tr}\left(Q_{2}\right)\left(\phi_{0}(s)\right) d s\right)\right]\right\} \\
& \times \operatorname{det}_{2}\left(\operatorname{Id}_{\mathcal{H}}+A\right)^{-1 / 2}
\end{aligned}
$$

Finally, by summarizing the above arguments, we can present the following theorem:

Theorem 4.9 Let $\alpha_{0}$ be denoted in Theorem 3.2. Then we have

$$
\begin{aligned}
\alpha_{0}= & \exp \left\{-\frac{1}{2} \operatorname{Tr}(A-\tilde{A})-D F\left(\phi_{0}\right)\left[\Gamma\left(\int_{0} b_{1}\left(\phi_{0}(s)\right)+\operatorname{Tr}\left(Q_{2}\right)\left(\phi_{0}(s)\right) d s\right)\right]\right\} \\
& \times \operatorname{det}_{2}\left(\operatorname{Id}_{\mathcal{H}}+A\right)^{-1 / 2} .
\end{aligned}
$$

\section{Examples}

\subsection{Heat processes on loop spaces}

In this subsection, we consider a class of stochastic processes on continuous loop spaces and show that the theory of rough paths is applicable to them. The processes are usually called heat processes on loop spaces and are defined by a collection of finite-dimensional SDEs. Processes of this kind were first introduced by Malliavin [18] in the case of loop groups and then were generalized by many authors.

Let $s>1 / 2$ and $\mathcal{L}_{0}\left(\mathbb{R}^{d}\right):=\left\{x \in C\left([0,1], \mathbb{R}^{d}\right) \mid x(0)=x(1)=0\right\}$. For $h \in \mathcal{L}_{0}\left(\mathbb{R}^{d}\right)$ of the form

$$
h(\tau)=\sum_{n \neq 0} \hat{h}(n)\left(e^{2 \pi \sqrt{-1} n \tau}-1\right),
$$

we set

$$
\|h\|_{H_{0}^{s}\left(\mathbb{R}^{d}\right)}^{2}:=\sum_{n \neq 0}|2 \pi n|^{2 s}|\hat{h}(n)|^{2}
$$

and $H_{0}^{s}\left(\mathbb{R}^{d}\right):=\left\{h \in \mathcal{L}_{0}\left(\mathbb{R}^{d}\right) \mid\|h\|_{H_{0}^{s}\left(\mathbb{R}^{d}\right)}<\infty\right\}$. It is well-known that $H_{0}^{s}\left(\mathbb{R}^{d}\right)$ is a Hilbert space embedded in $\mathcal{L}_{0}\left(\mathbb{R}^{d}\right)$ and that there exists a Gaussian measure $\mu^{s}$ such that the triplet $\left(\mathcal{L}_{0}\left(\mathbb{R}^{d}\right), H_{0}^{s}\left(\mathbb{R}^{d}\right), \mu^{s}\right)$ becomes an abstract Wiener space. When $s=1, \mu^{1}$ is the usual $d$-dimensional pinned Wiener measure and is of particular importance. For $\tau \in[0,1]$ and $j=1,2, \ldots, d$, we denote by $\delta_{\tau}^{j}$ the element in $\mathcal{L}_{0}\left(\mathbb{R}^{d}\right)^{*}$ defined by $\left\langle\delta_{\tau}^{j}, x\right\rangle=x^{j}(\tau)$ and set $x(\tau):=\left(x^{1}(\tau), \ldots, x^{d}(\tau)\right)$. Let $\left(w_{t}\right)_{t \geq 0}$ be a $\mathcal{L}_{0}\left(\mathbb{R}^{d}\right)$-valued Brownian motion associated with $\mu^{s}$. We set $w_{t}^{j}(\tau):=\left\langle\delta_{\tau}^{j}, w_{t}\right\rangle$ and $w_{t}(\tau):=\left(w_{t}^{1}(\tau), \ldots, w_{t}^{d}(\tau)\right)$. 
Now we give heat processes in a slightly generalized form. Let

$$
A_{j}(x)=\sum_{i=1}^{r} a_{i j}(x) \frac{\partial}{\partial x_{i}}, \quad A_{0}(x)=\sum_{i=1}^{r} b_{i}(x) \frac{\partial}{\partial x_{i}}, \quad V_{0}(x)=\sum_{i=1}^{r} \beta_{i}(x) \frac{\partial}{\partial x_{i}}
$$

be vector fields on $\mathbb{R}^{r}, j=1, \ldots, d$. We assume the following regularities on the coefficients:

$$
a_{i j}, b_{i}, \beta_{i} \in C_{b}^{4}\left(\mathbb{R}^{r}, \mathbb{R}\right) \quad \text { for } 1 \leq i \leq r, 1 \leq j \leq d .
$$

We write $a$ for the $r \times d$-matrix $\left\{a_{i j}\right\}_{1 \leq i \leq r, 1 \leq j \leq d}$ and write $b$ and $\beta$ for the column vectors $\left(b_{1}, \ldots, b_{r}\right)^{\mathrm{T}}$ and $\left(\beta_{1}, \ldots, \beta_{r}\right)^{\mathrm{T}}$, respectively.

For each fixed space parameter $\tau \in[0,1]$ and $\varepsilon>0$, we consider the following (finite dimensional) SDE:

$$
\begin{aligned}
d_{t} X_{t}^{\varepsilon}(\tau) & =\sum_{j=1}^{r} A_{j}\left(X_{t}^{\varepsilon}(\tau)\right) \circ \varepsilon d_{t} w_{t}^{j}(\tau)+A_{0}\left(X_{t}^{\varepsilon}(\tau)\right) \varepsilon^{2} d t+V_{0}\left(X_{t}^{\varepsilon}(\tau)\right) d t \\
& =a\left(X_{t}^{\varepsilon}(\tau)\right) \circ \varepsilon d_{t} w_{t}(\tau)+b\left(X_{t}^{\varepsilon}(\tau)\right) \varepsilon^{2} d t+\beta\left(X_{t}^{\varepsilon}(\tau)\right) d t
\end{aligned}
$$

with the initial data $X_{0}^{\varepsilon}(\tau)=0$. We will often write $X^{\varepsilon}(t, \tau):=X_{t}^{\varepsilon}(\tau)$. In Proposition 5.1 below, we will prove that $X^{\varepsilon}(t, \tau)$ has a bi-continuous modification. We call $X^{\varepsilon}=\left(X^{\varepsilon}(t, \cdot)\right)_{0 \leq t \leq 1}$ the heat process. $X^{\varepsilon}$ can be regarded as a random variable in $P\left(\mathcal{L}_{0}\left(\mathbb{R}^{d}\right)\right)$.

Next we recall that $\left(\mathcal{L}_{0}\left(\mathbb{R}^{d}\right), \mu^{s}\right)$ satisfies the exactness condition for all tensor norms (including the projective tensor norm) on $\mathcal{L}_{0}\left(\mathbb{R}^{d}\right) \otimes \mathcal{L}_{0}\left(\mathbb{R}^{d}\right)$. (See Lemma 4.1 in [10] for the proof.) Therefore the Brownian rough path $\bar{w} \in G \Omega_{p}\left(\mathcal{L}_{0}\left(\mathbb{R}^{d}\right)\right)$ defined by $\left(w_{t}\right)_{t \geq 0}$ exists and we can deal with our heat process $X^{\varepsilon}$ defined by (23) from the viewpoint of rough paths.

We define a Nemytski map $\tilde{\sigma}: \mathcal{L}_{0}\left(\mathbb{R}^{r}\right) \rightarrow L\left(\mathcal{L}_{0}\left(\mathbb{R}^{d}\right) \oplus \mathbb{R}^{2}, \mathcal{L}_{0}\left(\mathbb{R}^{r}\right)\right)$ by

$$
\tilde{\sigma}(y)\left[\left(x, u_{1}, u_{2}\right)\right](\tau):=a(y(\tau)) x(\tau)+b(y(\tau)) u_{1}+\beta(y(\tau)) u_{2}, \quad \tau \in[0,1] .
$$

for $\left(x, u_{1}, u_{2}\right) \in \mathcal{L}_{0}\left(\mathbb{R}^{d}\right) \oplus \mathbb{R}^{2}$ and $y \in \mathcal{L}_{0}\left(\mathbb{R}^{r}\right)$. Note that the assumption (22) implies $\tilde{\sigma} \in C_{b}^{4}\left(\mathcal{L}_{0}\left(\mathbb{R}^{r}\right), L\left(\mathcal{L}_{0}\left(\mathbb{R}^{d}\right) \oplus \mathbb{R}^{2}, \mathcal{L}_{0}\left(\mathbb{R}^{r}\right)\right)\right)$. Then we can consider a random element $\Phi\left(\iota\left(\overline{\varepsilon w}, \lambda^{\varepsilon}\right)\right)$ in $G \Omega_{p}\left(\mathcal{L}_{0}\left(\mathbb{R}^{r}\right)\right)$ through the differential equation in the rough path sense (2). The following proposition is taken from Lemma 4.8 in [10]. By this proposition, we can obtain a dynamics on $\mathcal{L}_{0}\left(\mathbb{R}^{r}\right)$ In the proof, the Wong-Zakai approximation theorem plays a crucial role.

Proposition 5.1 For each $\varepsilon>0,(t, \tau) \mapsto \Phi\left(\iota\left(\overline{\varepsilon w}, \lambda^{\varepsilon}\right)\right)_{1}(0, t)(\tau)$ is a bicontinuous modification of the two-parameter process $\left(X^{\varepsilon}(t, \tau)\right)_{0 \leq t \leq 1,0 \leq \tau \leq 1}$ defined in (23).

Remark 5.2 In [18], Kolmogorov's criterion is used for the proof of the existence of continuous modifications. Hence Proposition 5.1 is regarded as a revisit via the rough path theory. In this paper we assume that the starting 
loop is the constant loop at 0 for simplicity. However it is easy to modify the proof for general starting elements in $\mathcal{L}_{0}\left(\mathbb{R}^{r}\right)$ since the initial conditions may be arbitrary in the rough path theory. In other words, we do not need a Hölder-like condition on the starting loops.

We define the heat kernel measure $\nu_{t}$ to be the law of $X^{1}(t, \cdot)$ in the case of $V_{0}=0$. This measure $\nu_{t}$ is supported in $\mathcal{L}_{0}\left(\mathbb{R}^{r}\right)$. As a consequence of Theorem 3.1, we easily have a large deviation principle for $\nu_{t}$ by noting that $\left(X^{\varepsilon}(t, \cdot)\right)_{t \geq 0}$ and $\left(X^{1}\left(\varepsilon^{2} t, \cdot\right)\right)_{t \geq 0}$ have the same law under $V_{0}=0$. See the second item of Theorem 4.9 in [10] for details.

Theorem 5.3 Let $V_{0}=0$. Then the heat kernel measure $\nu_{t}$ satisfies a large deviation principle as $t \searrow 0$ with the good rate function $\tilde{I}$, where

$\tilde{I}(y)=\left\{\begin{array}{cc}\frac{1}{2} \inf \left\{\|\gamma\|_{\mathcal{H}\left(H_{0}^{s}\left(\mathbb{R}^{d}\right)\right)}^{2} \mid y=\Psi_{0}(\gamma)_{1}\right\}, \\ \text { if } y=\Psi_{0}(\gamma)_{1} \text { for some } \gamma \in \mathcal{H}\left(H_{0}^{s}\left(\mathbb{R}^{d}\right)\right), \\ \text { otherwise. }\end{array}\right.$

More precisely, for any measurable set $K \subset \mathcal{L}_{0}\left(\mathbb{R}^{r}\right)$, it holds that

$$
-\inf _{y \in K^{\circ}} \tilde{I}(y) \leq \liminf _{t \searrow 0} t \log \nu_{t}(K) \leq \limsup _{t \searrow 0} t \log \nu_{t}(K) \leq-\inf _{y \in \bar{K}} \tilde{I}(y) .
$$

Remark 5.4 Fang-Zhang [8] showed the large deviation principle for for heat processes and heat kernel measures on loop groups. Our Theorems 3.1 and 5.3 are regarded as generalizations of their results.

\subsection{SDEs on M-type 2 Banach spaces}

The theory of SDEs in infinite dimensional Hilbert spaces has been developed and is well understood. However, for general separable Banach spaces, there exist difficulties in defining a meaningful Itô's integral. Recently, Brzeźniak and Elworthy developed a theory of SDEs for a certain class of Banach spaces called M-type 2 Banach spaces. In this subsection, we consider SDEs on Mtype 2 Banach spaces. For detailed explanations and further references, we refer the reader to see Brzeźniak-Carroll [5] and Brzeźniak-Elworthy [6].

Let $(X, H, \mu)$ be an abstract Wiener space and $w=\left(w_{t}\right)_{t \geq 0}$ be the $X$ valued Brownian motion. We assume that $(X, \mu)$ satisfies the exactness condition for the project tensor norm on $X \otimes X$. Let $Y$ be a M-type 2 Banach space (see Definition 2.1 in [6] for the definition). For a progressively measurable process $\xi=\left(\xi_{t}\right)_{0 \leq t \leq 1}$ which takes values in $L(X, Y)$ and satisfies $\mathbb{E}\left[\int_{0}^{1}\left|\xi_{s}\right|_{L(X, Y)}^{p} d s\right]<\infty, p>1$, we can define the stochastic integral $I(t):=\int_{0}^{t} \xi_{s} d w_{s}, 0 \leq t \leq 1$, as a continuous $Y$-valued martingale. Moreover there exists a constant $c_{p}$, independent of $\xi$, such that

$$
\mathbb{E}\left[\sup _{0 \leq t \leq 1}\left|\int_{0}^{t} \xi_{s} d w_{s}\right|_{Y}^{p}\right] \leq c_{p}\left(\int_{0}^{1} \mathbb{E}\left[\left|\xi_{s}\right|_{L(X, Y)}^{2}\right] d s\right)^{p / 2}
$$


See Theorem 2.9 and Remark 2.11 in [6] for details.

Let $\sigma \in C_{b}^{4}(X, L(X, Y))$ and $b_{1}, b_{2} \in C_{b}^{4}(Y, Y)$. We consider the following Stratonovich type SDE on $Y$ :

$$
d X_{t}^{\varepsilon}=\sigma\left(X_{t}^{\varepsilon}\right) \circ \varepsilon d w_{t}+b_{1}\left(X_{t}^{\varepsilon}\right) \varepsilon^{2} d t+b_{2}\left(X_{t}^{\varepsilon}\right) d t, \quad X_{t}^{\varepsilon}=0 .
$$

Here we call $\left(X_{t}^{\varepsilon}\right)_{0 \leq t \leq 1}$ is a solution to (25) if and only if it satisfies for each $0 \leq t \leq 1$

$$
\begin{aligned}
X_{t}^{\varepsilon}=\int_{0}^{t} \sigma\left(X_{s}^{\varepsilon}\right) \varepsilon d w_{s}+ & \int_{0}^{t} \operatorname{Tr}\left(Q_{2}\right)\left(X_{s}^{\varepsilon}\right) \varepsilon^{2} d s \\
& +\int_{0}^{t} b_{1}\left(X_{s}^{\varepsilon}\right) \varepsilon^{2} d s+\int_{0}^{t} b_{2}\left(X_{s}^{\varepsilon}\right) d s, \quad \text { a.s.. }
\end{aligned}
$$

Under our conditions for the coefficients, there exists a unique solution $\left(X_{t}^{\varepsilon}\right)_{0 \leq t \leq 1}$ to the SDE (25). See Theorem 2.26 in [6] and Theorem 2 in [5] for the detail. Moreover they have already established the Wong-Zakai approximation theorem (Theorem 3 in [5]) for the SDE (25). Then by the same argument as in the previous subsection, we can obtain

Proposition 5.5 For each $\varepsilon>0, t \mapsto \Phi\left(\iota\left(\overline{\varepsilon w}, \lambda^{\varepsilon}\right)\right)_{1}(0, t)$ is almost surely equal to $\left(X_{t}^{\varepsilon}\right)_{0 \leq t \leq 1}$ defined in (25).

Remark 5.6 Here we explain why we do not consider heat processes in the previous subsection on the Sobolev-Slobodetski space $W^{\theta, p}\left(S^{1}\right)$. Note that $W^{\theta, p}\left(S^{1}\right)$ is an M-type 2 Banach space and is continuously embedded in the space of continuous loops if $p>1$ and $1 / p<\theta<1$. (See Section 5 of [6] for details.) In Section 6 of [6] and Section 4 of [5], it is proved that $\left(X_{t}^{\varepsilon}\right)_{t \geq 0}$ defined by the $S D E$ (23) can be considered as the solution of a $W^{\theta, p}\left(S^{1}\right)$-valued $S D E(25)$ if $p>2$ and $1 / p<\theta<1 / 2$ (at least for the case $s=1$ ).

For general $s>1 / 2$, under a suitable condition on $p$ and $\theta$, we see by straightforward computation that the Gaussian measure $\mu_{s}$ is supported on $W^{\theta, p}\left(S^{1}\right)$ and see from inequality (6.8) in page 575 of [15] that $W^{\theta, p}\left(S^{1}\right) \otimes$ $W^{\theta, p}\left(S^{1}\right)$ is exact with respect to $\mu_{s}$.

Hence, one may wonder why we do not work on the Sobolev-Slobodetski space $W^{\theta, p}\left(S^{1}\right)$. The main reason why we avoided $W^{\theta, p}\left(S^{1}\right)$ is that the map $\sigma$ in (24) is not bounded with respect to the topology of $W^{\theta, p}\left(S^{1}\right)$. (See Section 5 of [6].) Since we would not like to treat the Itô maps or ODEs with unbounded coefficients, we choose to work on the space of continuous loops.

Acknowledgment. The authors would like to thank Professors Shigeki Aida, Sergio Albeverio, David Elworthy, Paul Malliavin and Shinzo Watanabe for their helpful comments and encouragements. The second author is very grateful to the organizers of the Abel symposium 2005 for giving him opportunities to talk in the conference and to contribute in this volume. The authors were supported by JSPS Research Fellowships for Young Scientists and the second author is supported by 21st century COE program "Development of Dynamic 
Mathematics with High Functionality" at Faculty of Mathematics, Kyushu University.

\section{References}

1. S. Aida, Semi-classical limit of the bottom of spectrum of a Schrödinger operator on a path space over a compact Riemannian manifold, preprint, 2006.

2. R. Azencott, Formule de Talyor stochastique et développement asymptotique d'intégrales de Feynman, Seminar on Probability, XVI, Supplement, pp. 237285, Lecture Notes in Math., 921, Springer, Berlin-New York, 1982.

3. G. Ben Arous, Methods de Laplace et de la phase stationnaire sur l'espace de Wiener, Stochastics 25, (1988), no.3, pp. 125-153.

4. V.I. Bogachev, Gaussian Measures, American Mathematical Society, Providence, RI, 1998.

5. Z. Brzeźniak and A. Carroll, Approximations of the Wong-Zakai type for stochastic differential equations in M-type 2 Banach spaces with applications to loop spaces, Séminaire de Probabilités XXXVII, pp. 251-289, Lecture Notes in Math., 1832, Springer, Berlin, 2003.

6. Z. Brzeźniak and K. D. Elworthy, Stochastic differential equations on Banach manifolds, Methods Funct. Anal. Topology 6 (2000), no. 1, pp. 43-84.

7. J.D. Deuschel and D.W. Stroock, Large Deviations, Academic Press, Boston, 1989.

8. S. Fang and T.S. Zhang, Large deviations for the Brownian motion on loop groups, J. Theoret. Probab. 14 (2001), no. 2, pp. 463-483.

9. Y. Inahama, Laplace's method for the laws of heat processes on loop spaces, J. Funct. Anal. 232 (2006), pp. 148-194.

10. Y. Inahama and H. Kawabi, Large deviations for heat kernel measures on loop spaces via rough paths, J. London Math. Society 73 (2006), no. 3, pp. 797-816.

11. Y. Inahama and H. Kawabi, Asymptotic expansions for the Laplace approximations for Itô functionals of Brownian rough paths, To appear in J. Funct. Anal. (2007), doi:10.1016/j.jfa.2006.09.016, 53 pages.

12. H.H. Kuo, Gaussian Measures on Banach Spaces, Lecture Notes in Math., 463, Springer, Berlin, 1985.

13. S. Kusuoka and D.W. Stroock, Precise asymptotics of certain Wiener functionals, J. Funct. Anal. 99 (1991), no. 1, pp. 1-74.

14. S. Kusuoka and D.W. Stroock, Asymptotics of certain Wiener functionals with degenerate extrema, Comm. Pure Appl. Math. 47 (1994), no. 4, pp. 477-501.

15. M. Ledoux, T. Lyons and Z. Qian, Lévy area of Wiener processes in Banach spaces, Ann. Probab. 30 (2002), no. 2, pp. 546-578.

16. M. Ledoux, Z. Qian and T.S. Zhang, Large deviations and support theorem for diffusion processes via rough paths, Stochastic Process. Appl. 102 (2002), no. 2, pp.265-283.

17. T. Lyons and Z. Qian, System control and rough paths, Oxford University Press, Oxford, 2002.

18. P. Malliavin, Hypoellipticity in infinite dimensions, Diffusion processes and related problems in analysis, Vol. I (Evanston, IL, 1989), pp. 17-31, Progr. Probab., 22, Birkhäuser Boston, Boston, MA, 1990. 
19. M. Schilder, Some asymptotic formulas for Wiener integrals, Trans. Amer. Math. Soc. 125 (1966), 63-85.

20. S. Takanobu and S. Watanabe: Asymptotic expansion formulas of the Schilder type for a class of conditional Wiener functional integrations, in "Asymptotic problems in probability theory: Wiener functionals and asymptotics "(Sanda/Kyoto, 1990), pp. 194-241, Pitman Res. Notes Math. Ser., 284, Longman Sci. Tech., Harlow, 1993. 